03.1;04.1;09.1;15.1

\title{
Причины свечения аномального вторичного потока в сверхзвуковых кластированных струях, возбужденных высоковольтным электронным пучком
}

\author{
(C) К.А. Дубровин, А.Е. Зарвин ", В.В. Каляда, А.С. Яскин \\ Новосибирский государственный университет, Новосибирск, Россия \\ ฯ E-mail: zarvin@phys.nsu.ru
}

Поступило в Редакцию 9 августа 2019г.

В окончательной редакции 9 августа 2019г.

Принято к публикации 10 января 2020г.

\begin{abstract}
Установлена роль кластированных частиц в формировании вторичного потока („следа“), образующегося при истечении газа в разреженную среду в режимах конденсации. Найдены границы „следа“ по результатам сравнения спектральных и фотометрических измерений. Рассмотрены возможные механизмы инициации его свечения. Установлена роль энергообмена кластеров с фоновым газом в послесвечении „следа“. Определены длины волн и соответствующие переходы в нейтральных (Ar-I) и однократно ионизованных (Ar-II) aтомах аргона, обусловливающие аномальное свечение. Установлены времена жизни в возбужденном состоянии частиц в центральной части и на периферии кластированного потока. Обнаружено влияние конденсации на проникание фонового газа в первичную традиционную сверхзвуковую струю и в зону „следа“.
\end{abstract}

Ключевые слова: сверхзвуковой газовый поток, кластер, аномальное излучение, аргон.

DOI: 10.21883/PJTF.2020.07.49217.18011

Экспериментальное моделирование процессов истечения газов из сопел в вакуум или сильно разреженное пространство является актуальной и сложной научно-прикладной задачей [1-3]. Особую сложность при моделировании на малогабаритных установках течения из/вблизи сопел реальных космических аппаратов представляет воспроизведение условий истечения газов. В частности, использование холодных потоков вместо реальных приводит к образованию кластеров, что может вызвать в свою очередь изменение размеров и структуры [4] сверхзвуковой струи разреженного газа.

На рис. 1 приведены примеры визуализаций струй азота $(a)$ и аргона $(b)$, истекающих из сверхзвукового сопла. Ввиду резкого уменьшения числа частиц вниз по потоку визуализировать перепад плотности в несколько порядков фотографически затруднительно, поэтому на всех приведенных изображениях область течения разделена на ряд участков со скорректированной яркостью.

В струе азота (рис. 1, a) при среднем размере кластеров $\langle S\rangle \sim 90$ [5] видны традиционная веретенообразная структура первой „бочки“ [6], область смыкания боковых границ струи, а также размытые контуры второй „бочки“. В то же время в струе аргона (рис. $1, b ;\langle S\rangle \sim 1.5 \cdot 10^{3}$ ) помимо аналогичных структур обнаруживается внешняя светящаяся область (названная „следом“ [4]), также имеющая форму „бочки“, но со значительно бо́льшими размерами, сложной структурой и слабозатухающим свечением при возбуждении излучения электронным ударом. Предположительно возникновение этой структуры обусловлено присутствием в струе аргона кластеров большого размера.
Настоящее исследование направлено на изучение условий формирования „следа“ в струях с развитой конденсацией, рассмотрение возможных причин и механизмов инициации послесвечения „следа“, а также процесса проникания фонового газа внутрь струи. Работа выполнена на комплексе ЛЭМПУС-2 НГУ [7], обеспечивающем поддержание давления в фоновом пространстве в диапазоне $10^{-4}-10^{2} \mathrm{~Pa}$. Моделирование истечения сверхзвуковых струй проводилось с использованием сопел субмиллиметрового размера. Для визуализации течения в качестве источника возбуждения излучения был использован высоковольтный электронный пучок. В экспериментах применены известные методы фотометрии [8] и спектроскопии [9] потоков. Монтаж спектрального прибора на координатное устройство позволил проводить измерения как в области возбуждения излучения, так и за ее пределами.

Установлено, что „след“ не образуется за звуковыми соплами при доступных в исследовании начальных параметрах истечения, а также за сверхзвуковыми соплами при низком давлении торможения $P_{0}$. С увеличением $P_{0}$ размеры „следа“ увеличиваются, интенсивность свечения возрастает, а структура приобретает слоистый характер. Уменьшение давления в фоновом пространстве $P_{h}$ приводит к ослаблению интенсивности свечения и росту физических размеров „следа“. В условиях истечения сверхзвуковой струи в сильно разреженный фоновый газ визуализировать „след“ не удалось.

Рассмотрим наиболее характерные варианты реакций в кластированном потоке при взаимодействии с электронами: 1) процесс возбуждения мономеров струи; 2) диссоциативный процесс возбуждения кластера с испаре- 

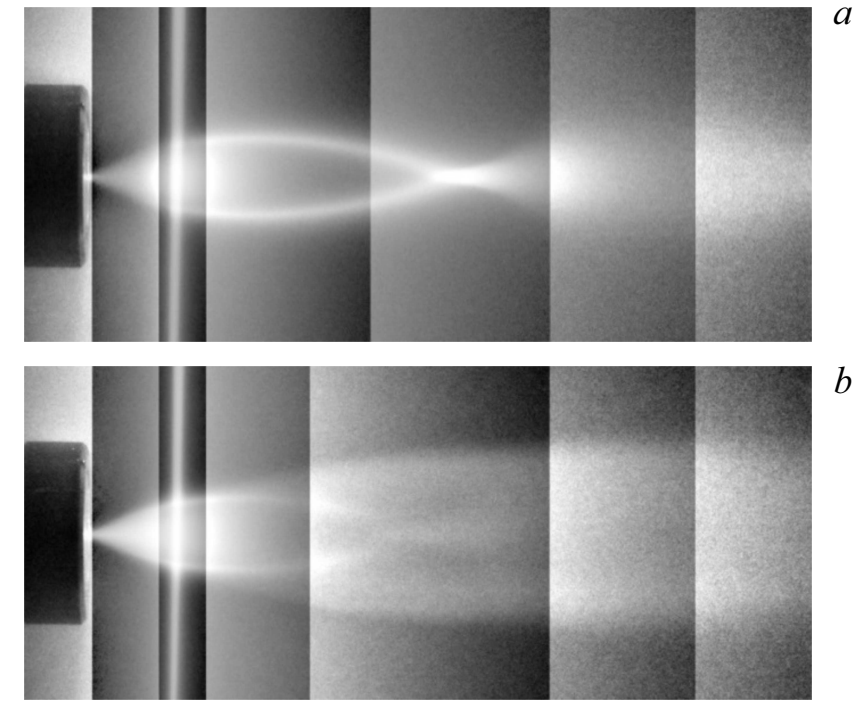

Рис. 1. Визуализация струй азота $(a)$ и аргона $(b)$, истекающих из сверхзвукового сопла (с диаметром критического сечения $d_{*}=0.256 \mathrm{~mm}$ ). Параметры истечения: $P_{0}=400 \mathrm{kPa}$, $P_{h}=4 \mathrm{~Pa}$.

нием возбужденного мономера; 3 ) процесс возбуждения долгоживущих уровней с фрагментацией кластера [10] при распаде экситонов и отсоединении возбужденной частицы (мономера, димера или тримера) [11]; 4) двухстадийный процесс столкновительного энергообмена с передачей энергии от возбужденного кластера атому или молекуле фонового газа; 5) трехстадийный процесс, включающий в себя передачу энергии кластеру путем присоединения возбужденного мономера фонового газа (или газа струи), внутрикластерный энергообмен и последующее испускание фотона одним из атомов кластера.

Ввиду того что частицы фонового газа вне пределов области смешения сверхзвуковой струи двигаются ненаправленно, вариант 1 не может являться причиной возникновения структуры „следа“.

В известных работах по прониканию фонового газа в сверхзвуковую струю $[6,12]$ не рассматривались режимы истечения с развитой конденсацией, т.е. в условиях формирования в потоке кластеров большого размера. В настоящей работе рассмотрена возможность механизма столкновения возбужденных кластеров с частицами фонового газа в качестве источника свечения в „следе“. Для проведения измерений использовалась методика, описанная в [12]. В качестве примеси при истечении сверхзвуковой струи аргона в пространство с фиксируемым давлением $P_{h}$ в фоновый газ (аргон) через боковой натекатель подмешивался молекулярный азот. Различием эффективности проникания разных газов (азота и аргона) вследствие различия их масс в данном случае пренебрегалось. Регистрация внутри потока аргона излучения азота, добавленного в фоновый газ, позволила определить степень проникания всего фонового газа $(p)$.
Зависимости проникания фонового газа внутрь струи аргона со „следом“ в поперечных сечениях струи на двух расстояниях от выходного среза сопла до электронного пучка $\left(X_{n(1)}\right.$ и $\left.X_{n(2)}\right)$ приведены на рис. 2, $a$. Регистрируемая интенсивность излучения азота (на пеpeходе $\mathrm{N}_{2}^{+}\left(B_{2} \Sigma_{u}^{+} \rightarrow X_{2} \Sigma_{g}^{+}\right)$, полосе 0-0, длина волны $\lambda=391 \mathrm{~nm}$ ) нормировалась на величину соответствующего сигнала в фоновом газе вдали от струи и пересчитывалась на суммарную долю проникающего газа с исключением вклада излучения газа струи (приведена в процентах). Визуализация данного режима приведена на рис. $2, b$. Поперечное сечение для $X_{n(1)}=42.3 \mathrm{~mm}$ (сплошная кривая) соответствует приблизительно середине первой „бочки“. Вне границ первой „бочки“ сигнал почти сразу достигает фонового значения (точка A) и падает до нуля на оси струи. Другой профиль при $X_{n(2)}=119.8 \mathrm{~mm}$ соответствует поперечному срезу внутри второй „бочки“. В этом сечении фоновый азот проникает до оси струи. Штриховая кривая данного профиля достигает фонового значения в точке $C$. Однако в точке $B$ имеется перегиб, который предположительно соответствует выходу измерений за границу второй „бочки“ в область „следа“. В соответствии с визуализацией (рис. $2, b)$ точка $C$ лежит за границей визуально наблюдаемого „следа“.

Таким образом, можно сделать вывод, что в области „следа“ столкновение частиц потока с фоновым газом также препятствует прониканию фонового газа в струю. Установленное рассеяние фонового газа на частицах
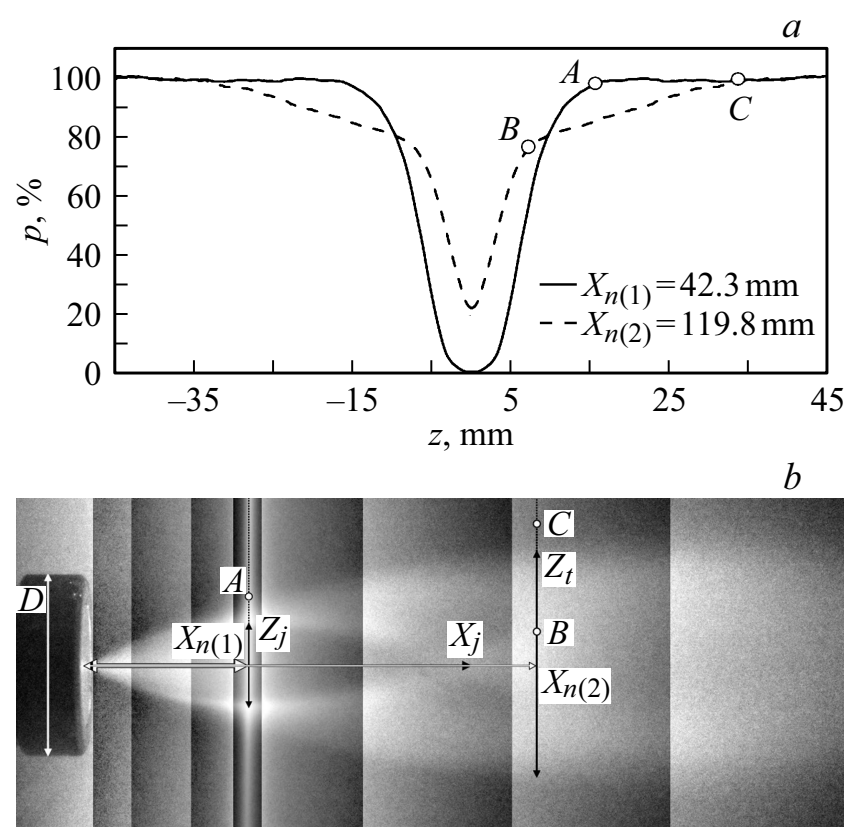

Рис. 2. Сравнение поперечных профилей доли проникающего фонового газа, зарегистрированных на разных расстояниях от оси струи $(a)$, в сопоставлении с визуализацией измерений $(b)$. Параметры истечения: $P_{0}=400 \mathrm{kPa}, P_{h}=2.93 \mathrm{~Pa}, \mathrm{Re}_{L}=61$, $\langle S\rangle=1.2 \cdot 10^{3} . X_{j}$ и $Z_{j}$ - продольный и поперечный размеры первой „бочки“, $Z_{t}-$ поперечный размер „следа“, $D$ диаметр шайбы сопла, $\operatorname{Re}_{L}$ - линейное число Рейнольдса. 

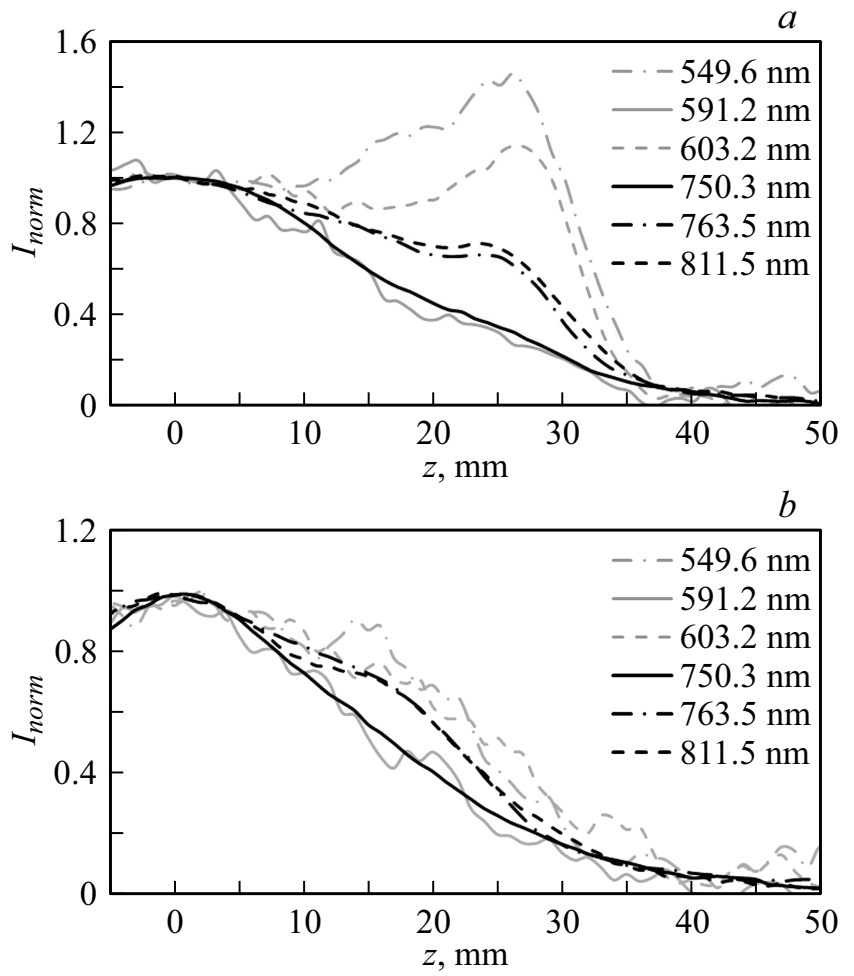

Рис. 3. Сравнение поперечных профилей нормированной интенсивности излучения, зарегистрированных на расстоянии сопло-электронный пучок 136.5 (a) и $241.5 \mathrm{~mm}(b)$ при смещении локализации измерений на $X_{k}=20 \mathrm{~mm}$ вниз по потоку.

Переходы, регистрируемые в серии измерений для атомарного аргона [13]

\begin{tabular}{c|c|c}
\hline$\lambda, \mathrm{nm}$ & $\tau_{k i}, \mathrm{~ns}$ & $A$, a.u. \\
\hline 549.6 & 591.7 & 25 \\
591.2 & 952.3 & 50 \\
603.2 & 406.5 & 70 \\
750.3 & 22.5 & $20 \cdot 10^{3}$ \\
763.5 & 40.8 & $25 \cdot 10^{3}$ \\
811.5 & 30.2 & $35 \cdot 10^{3}$
\end{tabular}

„следа““ позволяет предположить, что процессы 4 или 5 могут быть причинами возникновения наблюдаемого свечения.

Следует отметить, что в спектре аргона присутствует излучение как нейтральных (Ar-I), так и однократно ионизованных (Ar-II) атомов. Однако зарегистрированных линий переходов Ar-II очень мало, а их интенсивность излучения пренебрежимо мала по сравнению с интенсивностью излучения Ar-I.

Свечение в „следе“ наблюдается далеко за пределами области возбуждения потока. Для выяснения механизма этого свечения проведены измерения при смещении локальной области регистрации спектра излучения вниз по потоку на некоторое варьируемое расстояние $\left(X_{k}\right)$ от оси электронного пучка. В результате получены поперечные профили нормированной интенсивности $\left(I_{\text {norm }}\right)$ излучения отдельных линий аргона, зарегистрированные на расстоянии $X_{k}=20 \mathrm{~mm}$ (рис. 3). Регистрация профилей выполнена на наиболее интенсивных переходах нейтральных частиц аргона, параметры которых представлены в таблице $\left(\lambda-\right.$ длина волны, $\tau_{k i}-$ время жизни, а $A$ - вероятность указанного перехода).

На полученных профилях, соответствующих области „следа“ (что подтверждают результаты фотометрии), отслеживается особенность, проявляющаяся в увеличении интенсивности излучения вне границ первой и второй „бочек“. Обнаружено, что на нескольких длинах волн (штриховые линии разного вида) форма поперечных профилей имеет аномальный вид (второй максимум, расположенный на границе визуально наблюдаемого следа). В то же время на некоторых других длинах волн (сплошные линии разных оттенков серого) подобная особенность не обнаруживается. При сравнении табулированных времен жизни возбужденных состояний для переходов, соответствующих представленным длинам волн (см. таблицу), выявить закономерности не удалось.

С помощью измерений установлено, что переходы, визуализирующие границы „следа“, обладают в 2 раза бо́льшими временами жизни на первичном участке струи $\left(\tau_{(1)} \sim 7 \mu \mathrm{s}\right.$, для всех остальных переходов $\left.\tau_{(2)} \sim 3.4 \mu \mathrm{s}\right)$, причем данные времена для всех переходов увеличиваются при проведении измерений за пределами первой „бочки“.

Причиной увеличения времен жизни частиц в возбужденном состоянии (по сравнению с табличными значениями) могут служить процессы 2, 3 и 5. Однако при диссоциативных процессах 2 и 3 происходит эжекция метастабильных фрагментов с энергиями менее $1.5 \mathrm{eV}[11]$. Поэтому излучение фрагментов происходит в ИК-диапазоне, тогда как „след“ наблюдается в видимом. Следовательно, вероятность участия процессов 2 и 3 в излучении „следа“ крайне мала. Процесс 5 может являться причиной увеличения времен жизни в возбужденном состоянии в области „следа“, однако эти времена оказываются значительно больше и внутри изолированной от фонового газа первой „бочки“.

Таким образом, наиболее вероятной причиной свечения аномального вторичного потока в сверхзвуковых кластированных струях, возбужденных высоковольтным электронным пучком, является механизм взаимодействия кластеров „следа“, находящихся длительно в возбужденном состоянии вследствие внутрикластерного энергообмена, со статичными мономерными частицами фонового пространства - процесс 4.

\section{Финансирование работы}

Работа выполнена с использованием оборудования Центра коллективного пользования „Прикладная физика“ физического факультета НГУ при финансовой 
поддержке Российского фонда фундаментальных исследований (грант № 20-01-00332/20) и Министерства образования и науки РФ (гранты № 3.5918.2017/ИТР, 3.5920.2017/ИТР).

\section{Конфликт интересов}

Авторы заявляют, что у них нет конфликта интересов.

\section{Список литературы}

[1] Zischang J., Suhm M.A. // J. Chem. Phys. 2013. V. 139. N 2. P. 024201.

[2] Zhang S., Sobota A., Van Veldhuizen E.M., Bruggeman P.J. // J. Phys. D: Appl. Phys. 2014. V. 48. N 1. P. 015203.

[3] Korobeishchikov N.G., Penkov O.I. // Vacuum. 2016. V. 125. N 3. P. 205-208.

[4] Зарвин А.Е., Яскин А.С., Каляда В.В., Ездин Б.С. // Письма в ЖТФ. 2015. Т. 41. В. 22. С. 74-81.

[5] Hagena O.F., Obert W. // J. Chem. Phys. 1972. V. 56. N 5. P. 1793-1802.

[6] Кисляков Н.И., Ребров А.К., Шарафутдинов Р.Г. // ПМТФ. 1975. № 2. С. 42-52.

[7] Zarvin A.E., Kalyada V.V., Madirbaev V.Zh., Korobeishchikov N.G., Khodakov M.D., Yaskin A.S., Khudozhitkov V.E., Gimelshein S.F. // IEEE Trans. Plasma Sci. 2017. V. 45. N 5. P. 819-827.

[8] Poplavski S.V., Boiko V.M., Lotov V.V., Nesterov A.U. // J. Phys.: Conf. Ser. 2017. V. 894. P. 012115.

[9] Belan M., De Ponto S., Tordella D. // Exp. Fluids. 2008. V. 45. N 3. P. 501-511.

[10] Schütte S., Buck U. // Int. J. Mass Spectr. 2002. V. 220. N 2. P. 183-192.

[11] Van der Burgt P.J.M., McConkey J.W. // J. Chem. Phys. 1995. V. 102. N 21. P. 8414-8423.

[12] Кисляков Н.И., Ребров А.К., Шарафутдинов Р.Г. // ПМТФ. 1973. № 1. С. 121-127.

[13] Kazakov V.V., Kazakov V.G., Kovalev V.S., Meshkov O.I., Yatsenko A.S. // Phys. Scripta. 2017. V. 92. N 10. P. 105002. 